\title{
The Pathophysiologic Basis of Nuclear Medicine, Third Edition, Abdelhamid H. Elgazzar, Ed.
}

\author{
Springer International Publishing, Switzerland, 2015. ISBN 978-3-319-06111-5
}

\author{
Alessia Ruggiero $^{1} \cdot$ Luigi Mansi $^{1}$
}

Published online: 26 February 2016

(C) Springer-Verlag Berlin Heidelberg 2016

This book represents the third edition of The Pathophysiologic Basis of Nuclear Medicine, arriving after the success of the first two editions and of the synopsis published by Springer in 2014. The book is edited by Professor Abdelhamid H. Elgazzar, director of the Nuclear Medicine Department at Kuwait University and written in collaboration with many international experts, coming not only from his group, but also from leading institutions in the U.S., Egypt, Saudi Arabia and South Korea. The third edition has been widely updated compared to the previous edition published in 2006; it includes the most recent knowledge in nuclear medicine, and an improvement has also been made in iconography, reflecting the better quality produced by newer diagnostic tools and more appropriated acquisition techniques.

The title clearly individuates the content of the publication, representing an original contribution to the scenario of Nuclear Medicine publishing, in which very few texts are dedicated to pathophysiology. The book sets itself the task of clarifying mechanisms that underlie images in nuclear medicine, having as an aim a better understanding of the achievable information. This approach may certainly improve the use of different techniques, in terms of clinically directed reports and in research. The volume consists of 21 chapters and a final glossary, also expanded and updated from the previous edition. The 753 pages that constitute the publication are enriched by a large number of high-quality color images, all extensively commented on, and by explanatory tables elucidating the text. The first two chapters are introductory,

\section{Luigi Mansi}

luigi.mansi@unina2.it

1 Head and Chair of Nuclear Medicine, Second University of Naples, Piazza Miraglia, 80138 Napoli, Italy describing the concept of pathophysiology and providing a brief overview of molecular and cellular biology. The third chapter more specifically introduces the field of nuclear medicine, describing the general mechanisms that modulate the localization of radiopharmaceuticals. For this purpose, Tables 3.1, 3.2 and 3.3 are particularly useful, summarizing the most diffuse radiopharmaceuticals for diagnosis and therapy and their uptake mechanisms. Strictly clinical discussion begins with the fourth chapter, "Inflammation," which, after describing the general mechanisms underlying inflammatory processes, explores the topic of analyzing inflammatory phenomena related to the main organs and tissues. The core of the book consists of the next 15 chapters, which deal with the pathophysiologic bases of nuclear medicine, separately discussing each apparatus. All chapters have a similar format, consisting of a first part that describes the specific systemic pathophysiology, followed by a second part that describes the associated nuclear medicine procedures. The clinical applications of nuclear medicine therefore cover every major organ system and relate the basic knowledge of anatomy, physiology and pathology to the clinical utilization of various scintigraphic modalities. A special mention has to be given to chapters 11 and 12 , dedicated to nuclear oncology. In these pages, most important tumors are treated, beginning with phenomena underlying oncogenesis and then describing corresponding diagnostic techniques. The text ends with two chapters providing brief notes on radioiodine therapy and on the effects of ionizing radiations on human tissues, respectively.

The clear language, the wealth of images and tables, and the rigorous approach makes this text mandatory for residents in nuclear medicine, who may be stimulated to create solid bases in their future profession. Furthermore, the book is suitable for an audience of experts, as well as doctors, researchers and students who wish to approach the world of nuclear medicine. 